\title{
A Grid-based Unequal Clustering Algorithm for the Multi-hop Routing in WSN
}

\author{
YING XU \\ Key Laboratory for Embedded and \\ Network \\ Computing of Hunan Province \\ College of Information Science and \\ Engineering \\ Hunan University \\ Changsha, China \\ e-mail:hnxy@hnu.edu.cn
}

\author{
Xiongfei Zheng \\ Key Laboratory for Embedded and \\ Network \\ Computing of Hunan Province \\ College of Information Science and \\ Engineering \\ Hunan University \\ Changsha, China \\ e-mail:447236402@qq.com
}

\author{
Renfa Li \\ Key Laboratory for Embedded and \\ Network \\ Computing of Hunan Province \\ College of Information Science and \\ Engineering \\ Hunan University \\ Changsha, China \\ lirenfa@vip.sina.com
}

\begin{abstract}
Wireless sensor network consists of energy-constrained sensor nodes that are densely distributed in a monitoring area. Therefore, how to effectively use the energy of nodes to prolong the network lifetime becomes a key research topic. A grid-based unequal clustering algorithm for the multi-hop routing is proposed in this paper, in which the monitoring area is divided into virtual grids with different sizes. The algorithm provides a new estimation of the optimal number of grids. A cluster head selection method among the sensor nodes within the unique grid is adopted and modified. A multi-hop routing is designed for the communication between clusters. Simulation results show that the algorithm balances the energy consumption of the entire network and prolongs the network lifetime effectively.
\end{abstract}

\section{Keywords: Wireless Sensor Network, Routing .}

\section{INTRODUCTION}

Wireless sensor networks (WSN) has developed rapidly in recent years. With a very broad application prospects, it has a potential value in many different fields such as the military defense [1], the industrial and agricultural production [2], and the medical treatment [3], the things of internet [4], etc. However, sensor nodes have not only limited resources including the energy and the storage memory, but also limited processing and communication capabilities. How to balance energy consumption and extend the network lifetime have attracted much research attention. A large number of energy efficiency routing protocols and algorithms have thus proposed in the literature.

$\mathrm{LEACH}[5]$ is a typical WSN uniform clustering routing protocol, which uses the random clustering and periodically replaced the cluster heads. Cluster heads communicate with the base station using a single hop, which makes nodes far from the base station will consume too much energy. Meanwhile, the frequent clustering consumes more energy. Chengfa Li et al. [6] have designed an Energy-Efficient Unequal Clustering protocol (EEUC), utilizing the inter-cluster multi-hop to transmit data. In order to balance the energy consumption, the protocol uses a non-uniform competition radius to form the clusters different sizes, where clusters near the base station have small sizes. Zhumin et al. [7] have proposed a routing algorithm based on the virtual grids Clustering (CRVB). It divides the network area into virtual grids. A cluster head communicates with the base station via multiple hops and each cluster can communicate simultaneously. In CRVB, the cluster size is the same, which brings the problem called 'hot spot' due to the more energy consumption of the cluster heads near the base station which need to forward data from other cluster heads.

To solve the 'hot spot' problem, we propose a Grid-Based Unequal Clustering (GBUC) multi-hop routing algorithm for WSN. Firstly, based on the estimation of the optimal number for equal clusters proposed in literature [7], we extend it for the unequal clustering in this work; Secondly, the grid number Grid $_{\text {num }}$ of the network is set close to the estimated optimal number of clusters and an arithmetic sequence is used for the unequal clustering of the entire monitoring area; Thirdly, a unique cluster head selection method is used to select the cluster head; Each cluster head selects the least costly adjacent cluster head within their communication range as the next hop except its parent node. Finally, the inter-cluster multi-hop routing is adopted to save the energy.

The rest of the paper is organized as follows. In section 2 , we present the problem definition and the network model. Section 3 presents the proposed GBUC algorithm. We evaluate our algorithm by experiments and summarize the obtained results in section 4. Finally, section 5 concludes this paper.

\section{THE PROBLEM DEFINITION AND THE NETWORK MODEL}

The grid-based unequal clustering needs to address the following problems: 1) how to determine the optimal number of clusters; 2) how to calculate the size of the cluster; 3) how to select a unique cluster head ; 4) how to compute the multi-hop routing among the cluster heads.

We use the same wireless communication energy model and assumptions defined in literature [5]. Both the free space (with $d^{2}$ power loss) and the multi-path fading (with $d^{4}$ power loss) channel models are used in the model. The consumed energy of a node sending $k$ bits of data to another node with the distance $d$ is calculated as follows:

$$
E T x= \begin{cases}k \varepsilon_{\text {elec }}+k \varepsilon_{f s} d^{2} & d<d_{0} \\ k \varepsilon_{\text {elec }}+k \varepsilon_{m p} d^{4} & d \geqq d_{0}\end{cases}
$$


and to receive this message, the consumed energy is:

$$
E_{R x}=k \varepsilon_{e l e c}
$$

Where $d_{0}$ is a threshold distance and $\varepsilon_{\text {elec }}$ is the electronic energy. $\varepsilon_{f}$ is the free space module coefficient and $\varepsilon_{m p}$ is the multipath module coefficient.

\section{The GRID-BASED UNEQUCAL Clustering MULTI-HOP ROUTING ALGORITHM}

\section{A. The Virtual Grids Generation}

As shown in Figure 1, the algorithm divides the monitoring area into uneven grids, $N$ nodes randomly distribute in an $R \times R$ square area and nodes in the same grid join a cluster. The number of grids Grid $_{\text {num }}$ is decided by an estimated optimal number of clusters, which ensures the optimal number of clusters to cover the entire monitoring area. Grid $_{n u m}$ is defined as:

$$
\text { Grid }_{\text {num }}=\left(\left\lceil\sqrt{C_{\text {opt }}}\right\rceil\right)^{2}
$$

$C_{o p t}$ is the optimal number of clusters. $\left\lceil\sqrt{C_{o p t}}\right\rceil$ equals the nearest integer $m$ after rounding. The $\mathrm{X}$-axis and $\mathrm{Y}$-axis in the monitoring area are divided into $m$ parts according to an arithmetic sequence. Assuming the equal difference is $d$, we set the first item $a_{l}$ (the length of grid nearest to base station) is $R / 10 . R$ is the total side length. Then the $d$ can be calculated :

$$
\begin{aligned}
& a_{l}+\left(a_{1}+d\right)+\ldots+\left[a_{l}+(m-1) d\right]=R \\
& d=\frac{2\left(R-m a_{1}\right)}{m^{2}}
\end{aligned}
$$

The monitoring area is thus be divided into $m \times m$ unequal clusters, where the cluster size increases as the distance increases to the base station. Figure 1 shows the unequal clustering of the monitoring area.

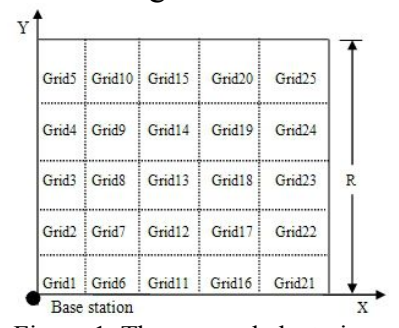

Figure 1. The unequal clustering.

Firstly, we need to estimate the optimal number of clusters

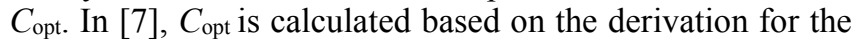
grids with an equal size. For the $R \times R$ square monitoring area, the total energy consumption of the network:

$$
\begin{gathered}
E_{\text {total }}=C E_{\text {head }}+N E_{\text {non-head }}=N k \varepsilon_{\text {elec }}+N k \varepsilon_{d a}+N l \varepsilon_{\text {elec }}+ \\
C k \varepsilon_{\text {mp }} d^{4} \text { toBS } \\
+N k \varepsilon_{f s} \frac{1}{2 \pi} \frac{R^{2}}{C}
\end{gathered}
$$

where $C$ is the number of equal clusters, $E_{\text {total }}$ is the total energy consumed by the network. $E_{\text {head }}, E_{\text {non-head }}$ represent energy consumed by the cluster head node and the non-cluster head, respectively. $\varepsilon_{\mathrm{da}}$ is the fusion rate. $k$ represents the number of packets sent in each round. $d_{t o B S}$ is the distance between the base station and the cluster head. After the first-order derivative of $C$ against $E_{\text {total }}$, the optimal cluster number $C_{\text {opt }}$ can thus be achieved:

$$
C_{o p t}=\frac{\sqrt{N}}{\sqrt{2 \pi}} \cdot \sqrt{\frac{\varepsilon_{\mathrm{fs}}}{\varepsilon_{m p}}} \cdot \frac{R}{d_{t o B S}^{2}}
$$

As for the unequal clustering, the above derivation cannot be directly used for the unequal clustering. We thus derivation the optimal clustering $C_{\mathrm{opt}}$ for the unequal clustering in this paper. For the unequal clustering, the number of nodes included in each cluster is different. When calculating the number nodes in each cluster, the parameter $d_{C i} / d_{\text {even }}$ should be added, where $d_{C i}$ is the distance between the cluster $i$ and the base station, $d_{\text {even }}$ is the average of all the distances between the geometric center of every cluster in the network and the base station.

$$
d_{\text {even }}=\frac{\sum_{i=1}^{n} d_{C i}}{C}
$$

The number of nodes in each cluster is $\frac{N}{C} \cdot \frac{d_{C i}}{d_{\text {even }}}$. Therefore, the energy consumption $E_{\text {head }}$ of the cluster head node in each round is:

$E_{\text {head }}=k \varepsilon_{\text {elec }}\left(\frac{N}{C} \frac{d_{C i}}{d_{\text {even }}}-1\right)+k \varepsilon_{d a} \frac{N}{C} \frac{d_{C i}}{d_{\text {even }}}+k \varepsilon_{\text {elec }}+k \varepsilon_{m p} d^{4}{ }_{i-B S}(9)$

where $d_{i-B S}$ is the distance between the cluster head $i$ and the base station. As the distance between the member node and the cluster head is relatively close, so the free space model in the above formula (1) is adopted. Then each member node's energy consumption $E_{\text {non-head }}$ is:

$$
E_{\text {non-head }}=k \varepsilon_{\text {elec }}+k \varepsilon_{f s} d^{2} \text { toHead }
$$

where $d_{\text {toHead }}$ represents the distance between a member node and the cluster head. The energy consumption of one cluster in each round $E_{\text {cluster }}$ is:

$$
E_{\text {cluster }}=E_{\text {head }}+\left(\frac{N}{C} \frac{d_{C i}}{d_{\text {even }}}-1\right) E_{\text {non-head }}
$$

Then the total energy $\mathrm{E}_{\text {total }}$ consumed by $C$ clusters is:

$$
\begin{aligned}
& E_{\text {total }}=E_{\text {clusterl } 1}+E_{\text {cluster } 2}+\cdots+E_{\text {clusterC } C} \approx 2 N k \varepsilon_{\text {elec }}+N k \varepsilon_{d a} \\
& +\left(N k \varepsilon_{f s}-C k \varepsilon_{f s}\right) d^{2}{ }_{\text {toHead }}+k \varepsilon_{m p} C d \frac{4}{c-B S}
\end{aligned}
$$


where $d_{\overline{c-B S}}$ represents the average distance from the cluster head to the base station. $d^{2}$ toHead can determined by its expectation [5]. Although the sizes of the cluster in this paper are inconsistent, but the expectation of $d^{2}$ toHead, the average is approximately equal, so $E\left[d^{2}{ }_{\text {toHead }}\right]$ in [5] can still be used.

$$
E\left[d^{2}{ }_{\text {toHead }}\right]=R^{2} /(2 \pi C)
$$

If the Eq. (13) is substituted in Eq. (12), then $C$ cluster consumes the total energy as:

$$
\begin{aligned}
& E_{\text {total }}=2 N k \varepsilon_{e l e c}+N k \varepsilon_{D A}+\left(N k \varepsilon_{f s}-C k \varepsilon_{f s}\right) R^{2} /(2 \pi C)- \\
& C k \varepsilon_{e l e c}+k \varepsilon_{m p} C d \frac{4}{c-B S}
\end{aligned}
$$

Then the first-order derivative of $C$ against $E_{\text {total }}$ in Eq. (14), i.e. $\frac{d E_{\text {total }}}{d C}=0$, then the $E_{\text {total }}$ can be achieved:

$$
C_{o p t}=\frac{\sqrt{N}}{\sqrt{2 \pi}} \cdot \sqrt{\frac{\varepsilon_{f S} R^{2}}{\varepsilon_{m p}\left(d \frac{4}{c-B S}-\varepsilon_{\text {elec }}\right)}}
$$

From the above derivation, it can be concluded that when the number of clusters is equal to the optimal number of clusters $C_{o p t}$, the energy consumption $E_{\text {total }}$ can be the minimum, namely the optimal network lifetime.

\section{B. The unique cluster head election method}

As the unique cluster head selection method in [6] does not take the circumstance of 0 cluster head, we make the improvement as follows:

(1)A random number between 0 and 1 is produced for each node in a cluster and compared with the threshold $T(N)$ [3], if it is less than the threshold, the node is selected as a candidate cluster head in the present round. $T(N)$ is calculated as follows:

$$
T(N)= \begin{cases}\frac{p}{1-p \times[r \bmod (1 / p)]} & N \in G_{\mathrm{r}} \\ 0 & N \notin G_{r}\end{cases}
$$

$p$ is the percentage of the cluster head number to the sum of all the nodes, $r$ is the present round number, and $G_{r}$ is the offset of member nodes which haven't been selected as the cluster head in previous rounds.

(2) If no candidate cluster head is found, then the nodes in the cluster with the remaining energy greater than 0 will be selected according to procedure (1). If this step repeats twice, without a candidate cluster head is found, then the cluster is regarded as the dead.

(3) If only one candidate remained, the node will be selected as the cluster head.

(4) If the number of candidates is greater than 1, then the node with the smallest random number is selected as the cluster head.

\section{The Multi-hop Routing}

The EEUC [6] protocol uses the multi-hop communication between clusters. Each cluster head $C_{i}$ chooses node of greater residual energy as the next hop node in two neighboring clusters of minimum relay distance in scope of competition of $C_{i}$. Relay distance function is defined as follows:

$$
d_{\text {relay }}^{2}=d_{i-j}^{2}+d_{j-B S}^{2}
$$

However it does not consider the effect of the number of nodes in neighboring clusters. For more member nodes, the cluster head consumes more energy. Besides, in EEUC, if the current node itself is selected as the next hop. This will lead to an infinitive loop of the data forwarding. Based on these observations, we improve the multi-hop routing as follows:(1) At first all cluster heads have no next hop and the status is marked as 0 . A variable distance threshold $T(d)$ as a competitive radius of each cluster head, $T(d)$ is defined [6]:

$$
T(d)=\left(1-c \frac{d_{\max }-d_{i-B S}}{d_{\max }-d_{\min }}\right) R_{c o m p}
$$

where $R_{\text {comp }}$ is the maximum competition radius. Because the cluster size is different in our GBUC, the maximum radius of the competition is also varies. We thus define it as the follows:

$$
T(d i)=\left(1-c \frac{d_{\max }-d_{i-B S}}{d_{\max }-d_{\min }}\right) R_{i}
$$

where $d_{\max }$ and $d_{\min }$ represent the maximum and minimum distance of a node in a cluster to a base station, respectively. $d_{i-B S}$ represents the distance of the current cluster head to the base station. $c$ is a constant factor between 0 and 1. $R_{i}$ $=\sqrt{x_{i}^{2}+y_{i}^{2}}$ represents the diagonal of a grid. According to Eq.(19), the competition radius changes between (1-c) $R_{i}$ and $R_{i}$.

(2) From those cluster heads within the scope of competition, we select three cluster heads with a minimal relay distance;

(3) From the selected three candidates, select two cluster heads with the maximal remaining energy;

(4) From the two candidates, select the node as the next hop with smaller member nodes. If the node is the current cluster head itself, the current cluster head forwards data directly to the base statin.

\section{THE SIMULATION EXPERIMENTS AND COMPARISONS}

\section{A. Simulation Environment}

We use Matlab to build the WSN simulation platform, all simulations run on a PC with one Intel i5 CPU of $2.5 \mathrm{GHz}$ on win 7 . We test the proposed algorithm with three different network sizes $(200,500$ and 1000). Parameters 
values are set as in the table.

Table1 Simulation Parameters

\begin{tabular}{|c|c|c|c|}
\hline Parameters & Value & Parameters & Value \\
\hline $\mathrm{N}$ & $200 / 500 / 1000$ & $\varepsilon_{\mathrm{da}} / \mathrm{J}$ & $5^{*} 0.000000001$ \\
\hline Probability p & 0.05 & $\varepsilon_{\text {elec }}$ & $50 \mathrm{~nJ} / \mathrm{bit}$ \\
\hline Network area & $(0,0) \sim(100,100)$ & $\varepsilon_{\mathrm{fs}}$ & $10 \mathrm{pJ} / \mathrm{bit} / \mathrm{m}^{2}$ \\
\hline Base station & $(0,0)$ & $\varepsilon_{\mathrm{mp}}$ & $0.0013 \mathrm{pJ} / \mathrm{bit} / \mathrm{m}^{4}$ \\
\hline $\mathrm{E}_{0} / \mathrm{J}$ & 0.5 & Packet size $/ \mathrm{bit}$ & 4000 \\
\hline
\end{tabular}

\section{B. Comparison of Experimental Results}

In this section, we compare GBUC with other two algorithms LEACH [5] and CRVB [7] in terms of the network lifetime and the energy consumption. As can be seen from Figure 2, the proposed GBUC algorithm prolongs the network lifetime effectively. More nodes in the same region, the better the performance obtained by GBUC compared to LEACH. The network with more nodes leads more grids, the energy consumed tends to be more balanced. On the contrary, the network with smaller size will has less grids, the size difference between two grids is also small, which makes GBUC is similar to the equal clustering CRVB.
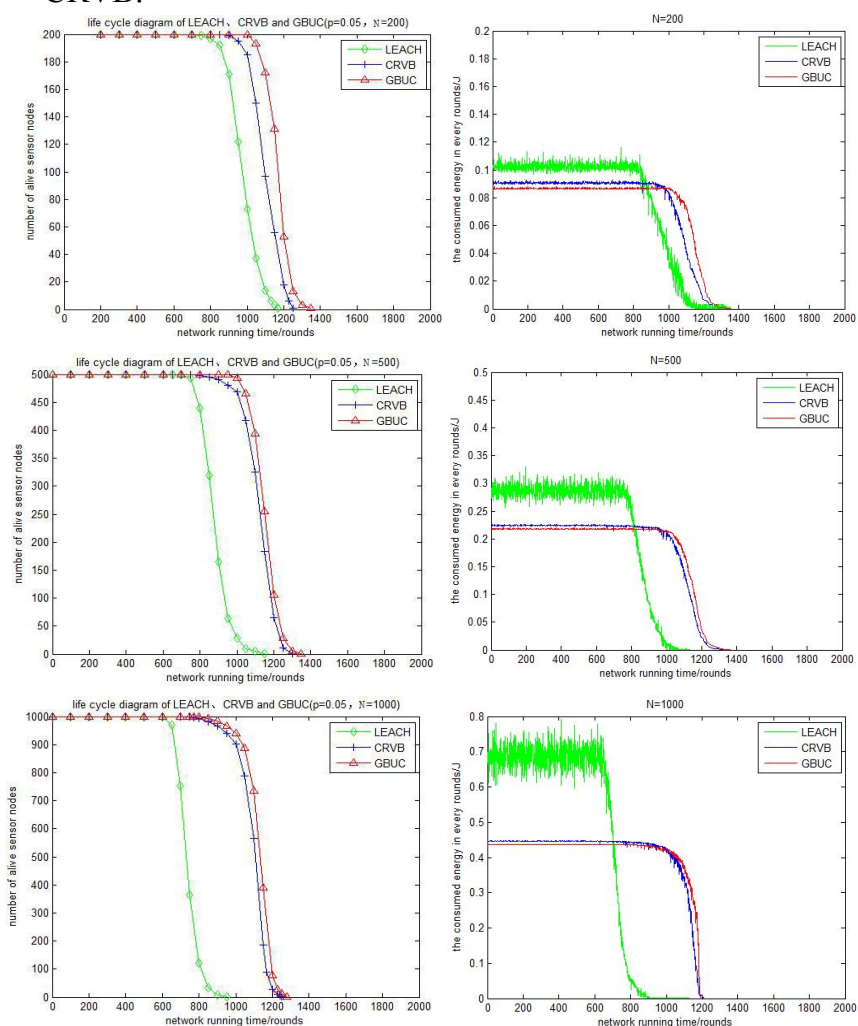

Figure2. The comparisons of the network lifetime and the energy consumption in each round for $N=200,500$ and 1000

The energy consumption per round determines the length of the network lifecycle. As can be seen from Figure 2, the total energy consumed per round of GBUC is lower than those of the other two algorithms. Both GBUC and CRVB are significantly better than LEACH. In summary, the GBUC algorithm performs better than LEACH and CRVB in terms of the network lifetime and the energy consumption.

\section{CONCLUSION}

In order to solve the 'hot spot' problem in WSN, a grid-based unequal clustering multi-hop routing algorithm GBUC has been proposed in this paper. In GBUC, we define a new expectation of the optimal clustering number. For the multi-hop routing, we consider not only the distance from neighborhood cluster heads to the base station and the residual energy, but also the node numbers in the cluster. Experimental results demonstrate the proposed GBUC can effectively balance the energy consumption and extend the lifetime of the network. This work can be extended for solving the dynamic routing problem in WSN.

\section{ACKNOWLEDGMENT}

This research is supported by Natural Science Foundation of China (NSFC project No. 61202289) and the project of the support plan for young teachers in Hunan University, China (Ref. 531107021137).

\section{REFERENCES}

[1] Horre W, Michiels S, Matthys N, "On the integration of sensor networks and general purpose IT infrastructruek," Proc. International Workshop on Middleware for Sensor Networks( IWMSN 02), 2007, pp.7-12.

[2] Kikuchi H, Iseki F, Moo W K. "Development of university network based on wireless ubiqutious network," Proc. International Conference on Advanced Communication Technology (ICACT 09), Feb. 2007, pp.189-194.

[3] Noury N, Here T, Rialle V. "Monitoring behavior in home using a smart fall sensor and postion sensors," Proc. IEEE-EMBS Special Topic Conference on Microtechnologies in Medicine and Biology. IEEE Computer Society, Jun. 2000, pp. 607-610.

[4] Gershenfeld N, Krikorian R, Cohen D. "The Internet of Things ," in Scientific American, 2004, pp. 76-81.

[5] Heinzelman W R, Chandrakasan A, Balakrishna H. "Energy-efficient communication algorithm for wireless micro sensor networks," Proc. Hawaii International Conference on System Sciences (HICSSS 33), IEEE Computer Society, Washington, 2002: 660-670.

[6] Chengfa Li, Mao Ye, Guihai Chen, "An Energy-Efficient Unequal Clustering Mechanism for Wireless Sensor Networks," Proc. IEEE International Conference. Mobile Ad-hoc and Sensor Systems Conference (MASS 02), Oct. 2005.

[7] Zhu Min, Xiao Zhen, Liu Haolin, Gao Hongbo, "A Routing Algorithm Based on Virtual Grid in WSN," in Journal of Sichuan University, vol. 44, May. 2012, pp. 143-148.

[8] Wang Jiangtao, Yang Geng, Chen Shengshou, "Secure LEACH Routing Protocol Based on Optimal Number of Head Nodes for Wireless Sensor Network," in Journal of Nanjing University of Posts and Telecommunications. vol. 28, Mar. 2008, pp. 27-32.

[9] Xu Jingqi, Wang Min. "A Dynamic Clustering Routing Protocol for Grid-Based Wireless Sensor Networks," in Computer Measurement \& Control, vol. 20, Jun. 2012, pp. 1729-1732.

[10] Akyildiz I F, Su W, Sankarasubramaniam Y, "Wireless sensor networks: a survey ," in Computer Networks, vol. 38, Apr. 2002, pp. 393-422.

[11] Robert Akl, Uttara Sawant. "Grid-based Coordinated Routing in Wireless Sensor Networks," Proc. Consumer Communications and Networking Conference (CCNC), Las Vegas, Nv, USA, Jul. 2007, pp. 800-864. 